\title{
What is a "good" result after transcatheter mitral repair? Impact of $2+$ residual mitral regurgitation
}

\author{
Nicola Buzzatti, MD, ${ }^{\mathrm{a}}$ Michele De Bonis, MD, ${ }^{\mathrm{a}}$ Paolo Denti, MD, ${ }^{\mathrm{a}}$ Fabio Barili, MD, ${ }^{\mathrm{b}}$ Davide Schiavi, BS, \\ Giovanna Di Giannuario, MD, ${ }^{\mathrm{a}}$ Giovanni La Canna, MD,${ }^{\mathrm{a}}$ and Ottavio Alfieri, $\mathrm{MD}^{\mathrm{a}}$
}

\section{ABSTRACT}

Objective: The study objective was to assess the impact on follow-up outcomes of residual mitral regurgitation $2+$ in comparison with $\leq 1+$ after MitraClip (Abbott Vascular Inc, Santa Clara, Calif) repair.

Methods: We compared the outcomes of mitral regurgitation $2+$ and mitral regurgitation $\leq 1+$ groups among a population of 223 consecutive patients with acute residual mitral regurgitation $\leq 2+$ who underwent MitraClip implantation at San Raffaele Scientific Institute (Milan, Italy) between October 2008 and December 2014.

Results: Residual mitral regurgitation $2+$ was found in 64 patients $(28.7 \%)$. Overall actuarial survival was $63.1 \% \pm 4.4 \%$ at 48 months. Cumulative incidence functions of cardiac death in patients with mitral regurgitation $2+$ was significantly higher (Gray test $P<.001$ ) compared with the mitral regurgitation $\leq 1+$ group. The adjusted hazard ratio was 5.28 (95\% confidence interval, 2.41-11.56, $P<.001)$. Cumulative incidence function of mitral regurgitation $\geq 3+$ recurrence in patients with residual mitral regurgitation $\leq 1+$ and mitral regurgitation $2+$ at 48 months was $13.3 \% \pm 3.8 \%$ and $45.2 \% \pm 6.8 \%$, respectively (Gray test $P<.001$ ). Multivariate model showed that mitral regurgitation $2+$ was the only factor associated with the development of mitral regurgitation $\geq 3+$ at follow-up (adjusted hazard ratio, 6.71; $95 \%$ confidence interval, 3.48-12.90; $P<.001)$. Mitral regurgitation cause was not associated with cardiac death and recurrence of mitral regurgitation $\geq 3+$ at follow-up. No relationship between New York Heart Association class and followup time after MitraClip implant was found (odds ratio, 1.07; 95\% confidence interval, $0.98-1.15 ; P=.11)$, and factors related to postoperative New York Heart Association also included residual mitral regurgitation $2+(P=.07)$.

Conclusions: Residual $2+$ mitral regurgitation after MitraClip implantation was associated with worse follow-up outcomes compared with $\leq 1+$ mitral regurgitation, including survival, symptom relief, and mitral regurgitation recurrence. Better efficacy should be pursued by transcatheter mitral repair technologies. (J Thorac Cardiovasc Surg 2016;151:88-96)

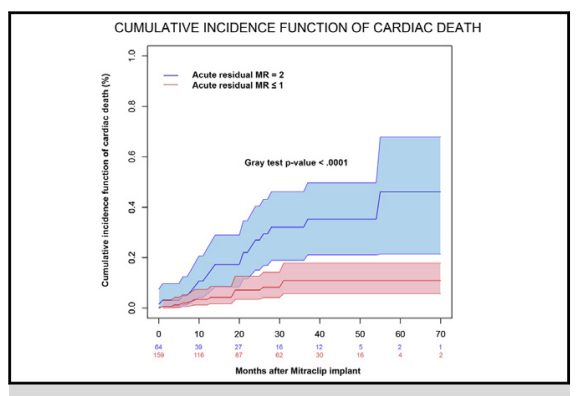

The CIF of cardiac death in patients with $2+$ versus $\leq 1+$ residual $M R$.

\section{Central Message}

Residual 2+ MR after MitraClip implantation was associated with worse survival, symptom relief, and MR recurrence compared with $\leq 1+$ MR.

\section{Perspective}

Residual 2+ MR is frequent after MitraClip (Abbott Vascular Inc, Santa Clara, Calif) implantation. In our series, it was associated with worse survival, symptom relief, and MR recurrence compared with the $\leq 1+$ MR group. Better efficacy should be pursued by transcatheter mitral repair technologies, especially before expanding indications to lower-risk patients.

See Editorial Commentary page 97.

See Editorial page 7.
Transcatheter mitral repair with the MitraClip system (Abbott Vascular Inc, Santa Clara, Calif) has emerged in recent years as a valid option to treat patients affected by

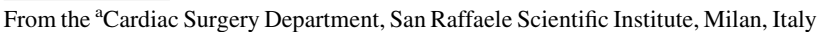
and ${ }^{\mathrm{b}}$ Cardiac Surgery Department, Santa Croce Hospital, Cuneo, Italy.

Read at the 95th Annual Meeting of The American Association for Thoracic Surgery, Seattle, Washington, April 25-29, 2015.

Received for publication April 2, 2015; revisions received Sept 7, 2015; accepted for publication Sept 10, 2015; available ahead of print Nov 4, 2015.

Address for reprints: Nicola Buzzatti, MD, via Olgettina 60, 20132 Milan, Italy (E-mail: buzzatti.nicola@hsr.it).

$0022-5223 / \$ 36.00$

Copyright (c) 2016 by The American Association for Thoracic Surgery

http://dx.doi.org/10.1016/j.jtcvs.2015.09.099 severe mitral regurgitation (MR) who are high risk for conventional surgery or inoperable ${ }^{1}$ and who would otherwise be left untreated. ${ }^{2}$ In this high-risk setting, the MitraClip system demonstrated excellent acute safety results at the price of significant rates of residual MR. . $^{3,4}$

Although it is well recognized by now that more than moderate residual MR after MitraClip implantation is associated with impaired follow-up outcomes, ${ }^{5-7}$ data on the long-term impact of residual $2+$ MR are still scarce. ${ }^{5,8}$ Residual $2+$ MR is still often considered as a procedural success and is present in approximately $40 \%$ of all patients treated with the MitraClip at the end of the procedure. $^{3,9}$ Of note, in the surgical setting, $2+$ residual 


\section{Abbreviations and Acronyms \\ $\mathrm{CI}=$ confidence interval \\ $\mathrm{CIF}=$ cumulative incidence function \\ DMR $=$ degenerative mitral regurgitation \\ FMR = functional mitral regurgitation \\ HR = hazard ratio \\ LVAD $=$ left ventricular assist device \\ MR = mitral regurgitation \\ NYHA $=$ New York Heart Association}

MR is well known to be associated with increased long-term recurrence of severe MR. ${ }^{10}$ This topic is particularly of interest, especially when assessing the convenience to expand transcatheter mitral repair procedures to intermediate- or low-risk patients.

The purpose of this study is to compare the follow-up outcomes of patients with residual $2+$ MR after the MitraClip procedure with those of patients with residual $\leq 1+$ MR from our high-volume single-center experience.

\section{MATERIALS AND METHODS}

From October 2008 to December 2014, a total of 243 consecutive patients affected by MR $\geq 3+$ were treated with the MitraClip at San Raffaele Scientific Institute (Milan, Italy). All patients were still symptomatic despite optimal medical therapy and deemed to be high risk for surgery or inoperable after discussion by the heart team. Surgical risk was assessed using logistic European System for Cardiac Operative Risk Evaluation, Society of Thoracic Surgeons Predicted Risk Of Mortality, and other features not captured by the mentioned risk scores (eg, frailty, porcelain aorta, thoracic graft patency). All patients underwent a standardized prospective data-collection pathway including preoperative transesophageal echocardiographic assessment. Although Endovascular Valve Edge-to-Edge REpair Study II eligibility criteria were used as a reference, ${ }^{11}$ a number of patients outside these were also treated and included in the present study. After the procedure, all patients were enrolled in our dedicated echocardiographic outpatient clinic, undergoing a transthoracic echocardiogram at every visit and being kept on optimal medical therapy.

MR was graded through echocardiography as $1+=$ mild, $2+=$ moderate, $3+=$ medium or moderate-to-severe, and $4+=$ severe. Because of the potential inaccuracy of conventional parameters for MR quantification in the setting of subverted mitral valve anatomy (double orifice), the jet-related parameters (vena contracta, effective regurgitant orifice) were used only in the presence of a single-orifice regurgitant jet, although the regurgitant fraction (difference between mitral inflow and aortic outflow stroke volume) ${ }^{12}$ was used in multiple-jet MR. Patients who did not present to our outpatient clinic were reached by telephone calls and asked to provide a recent $(<6$ months) echocardiogram.

Patients with acute procedural failure (residual MR $3+$ and $4+$ ) were excluded from this study. Follow-up outcomes of patients with acute residual $2+$ MR were compared with those of patients with residual $\leq 1+$ MR. In addition to those from the sample, outcomes from the functional MR (FMR) and degenerative MR (DMR) groups were separately analyzed and reported. Data-collection time was closed and analysis was performed in March of 2015. Ethics approval for the study was obtained from the ethics committee of our institution.

\section{Statistical Analysis}

Statistical analysis was conducted using SPSS Statistics software version 20 (IBM Corp, Armonk, NY) and R 3.2.1 software (R Development Core Team 2015. R: A language and environment for statistical computing. R Foundation for Statistical Computing, Vienna, Austria. ISBN 3-900051-07-0, available at: http://www.R-project.org/). The distribution of variables was evaluated using the 1-sample KolmogorovSmirnov test. Continuous variables are presented as mean \pm sample standard deviation for data with a normal distribution or as median (25th and 75th percentile limits in brackets) for data with a non-Gaussian distribution. Categoric variables are expressed as proportions. Univariable comparisons have been performed using the paired $t$ test for normally distributed data. For non-normally distributed data, the Wilcoxon signed-rank test was used for paired continuous variables, and the Mann-Whitney test was used for unpaired continuous variables. The chi-square test was used for categoric data, and the Fisher exact test was used when the minimum cell size requirements for the chi-square test were not satisfied.

Kaplan-Meier estimates were used for analyzing long-term survival. Fine and Gray models were used in competing risk analysis for time to cardiac death, with noncardiac death as the competing risk and time to MR 3+ or greater recurrence, with death as competing risk. The selection of variables for the Fine and Gray models was performed by a forward stepwise regression with the Bayesian information criterion as selection criteria. Nonparametric analyses of the outcome variables of interest were computed with the cumulative incidence function (CIF), and subdistribution hazards and comparisons were computed with the Fine and Gray test. Direct regression modeling of the effect of covariates on CIF was performed through the semiparametric proportional hazard model for the subdistribution hazards proposed by Fine and Gray, allowing for time-varying effect of the covariates. Hazards proportionality and time-dependent effects were checked with the analysis of Shoenfeld residuals, Kolmogorov-Smirnov test, and Cramer von Mises test. ${ }^{13-15}$

New York Heart Association (NYHA) class changes over time were analyzed as longitudinal data by generalized linear mixed models, including random effects. ${ }^{16}$ Missing values occurred in only 7 preoperative variables, with a maximum percentage of missing values of $1.2 \%$. Missing values were substituted by single conditional mean imputation as described to reduce bias and increase statistical power. ${ }^{17,18}$

\section{RESULTS}

\section{In-Hospital Outcomes}

Residual MR $\leq 2+$ after the procedure was observed in 223 of 243 patients $(91.8 \%)$. Residual MR $2+$ was found in $43 / 155(27.7 \%)$ of patients affected by FMR vs $21 / 68$ $(30.9 \%)$ of those affected by DMR $(P=.63)$. (Table 1$)$. The major preoperative data features are shown in Table 2.

In the MR 2+ group, one single clip was implanted in 9 $(14.1 \%)$ patients, 2 clips were implanted in $44(68.7 \%), 3$ clips in $10(15.6 \%)$ and 4 clips in 1 patient $(1.6 \%)$. By comparison, in the $\mathrm{MR} \leq 1+$ group the number of implanted clips was as follows: 1 in $62(39.0 \%)$ patients, 2 in $94(59.1 \%), 3$ in $3(1.9 \%)$ and 4 in 0 patients. The number of clips implanted in the two groups was significantly different $(P<.001)$.

Thirty-day mortality was $1.8 \%$ (4/223): 1 postoperative multiorgan failure, 1 postoperative acute pulmonary bleeding due to emphysematous lung rupture, 1 pneumonia with superimposed heart failure, and 1 sudden death. 
TABLE 1. Acute postprocedural residual mitral regurgitation

\begin{tabular}{|c|c|c|c|}
\hline \multirow[b]{2}{*}{ MR grade } & \multicolumn{2}{|c|}{ MR type } & \multirow[b]{2}{*}{$P$ value } \\
\hline & Functional $(n=155)$ & Degenerative $(n=68)$ & \\
\hline 0 & $14(9.0)$ & $14(20.6 \%)$ & \\
\hline $1+$ & $98(63.2)$ & $33(48.5 \%)$ & \\
\hline $2+$ & $43(27.8)$ & $21(30.9 \%)$ & \\
\hline
\end{tabular}

$\overline{M R}$, Mitral regurgitation.

\section{Follow-up Outcomes}

Follow-up was $100 \%$ complete. Median follow-up time was 20.5 months (interquartile range, 8-36, up to 75 months). During follow-up, a total of 61 deaths occurred, with an overall actuarial survival of $74.4 \% \pm 3.4 \%$ at 24 months and $63.1 \% \pm 4.4 \%$ at 48 months (Figure 1, $A$ ). Figure $1, B$ reports the CIFs of cardiac death in patients with MR $\leq 1+(7.1 \% \pm 2.3 \%$ at 24 months and $10.9 \% \pm$ $3.1 \%$ at 48 months $)$ and with MR $2+(26.9 \% \pm 6.6 \%$ at 24 months and $35.3 \% \pm 7.4 \%$ at 48 months), with noncardiac death as competing risk. The difference between MR $\leq 1+$ and MR $2+$ was significant (Gray test $P<.001$ ). The unadjusted hazard ratio (HR) for time to cardiac death was 4.09 (95\% confidence interval $[\mathrm{CI}], 2.00-8.38 ; P<.001)$, with residual $\mathrm{MR} \leq 1+$ as the reference group. After adjusting for significant variables listed in Table 3 , the adjusted HR was 5.28 (95\% CI, 2.41-11.56; $P<.001)$ (Figure 1,C). The assumption of hazard proportionality was considered fulfilled on the basis of the analysis of Shoenfeld residuals and the tests for time-invariant effect (Kolmogorov-Smirnov $P=.15$, Cramer von Mises $P=.10)$. Other significant factors associated with cardiac death at follow-up were preoperative enddiastolic diameter, preoperative NYHA class IV, and estimated glomerular filtration rate less than $60 \mathrm{~mL} / \mathrm{kg} / \mathrm{min}$. The cause of MR was not associated with cardiac death in the model.

During follow-up, 10 patients $(4.5 \%)$ underwent mitral surgery (7 mitral valve replacements, 2 heart transplantations, 1 left ventricular assist device [LVAD]) and 3 patients $(1.3 \%)$ underwent a MitraClip reimplantation.

CIF of $\mathrm{MR} \geq 3+$ recurrence in patients with residual MR $\leq 1+(5.6 \% \pm 1.9 \%$ at 24 months and $13.3 \% \pm$ $3.8 \%$ at 48 months $)$ and residual MR $2+(45.2 \% \pm$ $6.8 \%$ at 24 months and $45.2 \% \pm 6.8 \%$ at 48 months), with death as competing risk, is shown in Figure 2, A. The difference between MR $\leq 1+$ and MR $2+$ was significant (Gray test $P<.001$ ). After adjusting for variables of Table 2 significantly associated with the event in univariate analysis, residual MR $2+$ after the procedure was the only significant factor related to the development of MR $\geq 3+$, with an adjusted HR of 6.71 (95\% CI, 3.48$12.90 ; P<.001$ ) (Figure 2, B). The assumption of hazard proportionality was considered fulfilled on the basis of the analysis of Shoenfeld residuals and the tests for time-invariant effect (Kolmogorov-Smirnov $P=.34$, Cramer von Mises $P=.27$ ). Even in this analysis, MR cause was not associated with the incidence of MR $\geq 3+$ at follow-up.

Preoperative NYHA class was significantly higher compared with follow-up data $(P<.001)$. Generalized linear mixed model for evaluating changes over time of NYHA as ordinal data demonstrated that there is no relationship between NYHA class and time after MitraClip implant (odds ratio, $1.07 ; 95 \% \mathrm{CI}, 0.98-1.15 ; P=.11$ ) and that factors related to follow-up NYHA were estimated glomerular filtration rate greater than $60 \mathrm{~mL} / \mathrm{kg} / \mathrm{min}$ and preoperative NYHA class. Moreover, residual MR 2+ after implantation was found to be related to follow-up NYHA class $(P=.07)$ (Table 4).

\section{Functional Mitral Regurgitation Group}

In the FMR group, 27 of 46 deaths (58.7\%) were cardiac related. CIFs of cardiac death with noncardiac death as competing risk were $8.7 \% \pm 3.0 \%$ at 24 months and $13.7 \% \pm 4.0 \%$ at 48 months in patients with MR $\leq 1+$ and $29.8 \% \pm 8.2 \%$ at 24 months and $48.9 \% \pm 9.7 \%$ at 48 months in patients with MR $2+$ (Figure $3, A$ ). The difference between MR $\leq 1+$ and MR $2+$ was significant (Gray test $P<.001$ ). The unadjusted HR for time to cardiac death was 4.54 (95\% CI, 2.15-9.61; $P<.001)$, with residual MR $\leq 1+$ as the reference group. The adjusted HR was superimposable to that of the general model (adjusted HR, 4.07; 95\% CI, 1.61-10.24; $P<.003$ ), and other factors were the same as reported in Table 3.

MR $\geq 3+$ recurrence at follow-up was observed in 28 of 155 patients $(18.1 \%)$. Of note, 6 of 28 cases $(21.4 \%)$ of MR recurrence occurred in the first 30 days after the procedure. Partial clip detachment was observed in 3 patients (all with $2+\mathrm{MR}$ ). CIF of MR $\geq 3+$ recurrence in patients with residual $\mathrm{MR} \leq 1+(5.0 \% \pm 2.2 \%$ at 24 months and $13.2 \% \pm 8.4 \%$ at 48 months) and residual MR $2+$ $(45.7 \% \pm 4.5 \%$ at 24 months and $45.7 \% \pm 8.4 \%$ at 48 months), with death as competing risk, is shown in Figure 3, B. The difference between $\mathrm{MR} \leq 1+$ and MR $2+$ was significant (Gray test $P<.001$ ). Regression modeling of the effect of covariates on CIF demonstrated that residual MR $2+$ after the procedure was the only factor significantly related to the development of $\mathrm{MR} \geq 3+$ (HR, 7.27; 95\% CI, 3.34-15.80; $P<.001$ ).

At last follow-up, NYHA class improved in both MR 2+ $(P<.001)$ and $\mathrm{MR} \leq 1+(P<.001)$ groups compared with baseline (paired data available for $141 / 155$ patients [91.0\%]), but class II or less was found in 27 of 38 patients $(71.0 \%)$ with MR 2+ versus 94 of 103 patients $(91.3 \%)$ with $\mathrm{MR} \leq 1+(P=.002)$. 
TABLE 2. Major preoperative patient characteristics

\begin{tabular}{|c|c|c|c|c|c|c|}
\hline \multirow[b]{2}{*}{ Characteristic } & \multicolumn{3}{|c|}{ FMR } & \multicolumn{3}{|c|}{ DMR } \\
\hline & $\mathbf{M R} \leq \mathbf{1}+(\mathbf{n}=\mathbf{1 1 2})$ & MR 2 $+(n=43)$ & $P$ value & $\mathbf{M R} \leq \mathbf{1}+(\mathbf{n}=\mathbf{4 7})$ & MR 2 $+(\mathbf{n}=\mathbf{2 1})$ & $P$ value \\
\hline \multicolumn{7}{|l|}{ Clinical } \\
\hline Age, y & $69.0 \pm 9.6$ & $70.7 \pm 8.6$ & .30 & $76.2 \pm 9.2$ & $76.7 \pm 14.1$ & .85 \\
\hline Male gender, $\mathrm{n}(\%)$ & $92(82.1)$ & $36(83.7)$ & .82 & $21(44.7)$ & $19(90.5)$ & $<.01$ \\
\hline logistic euroSCORE, \% median (IQR) & $17.1(7.3-28.2)$ & $22.0(12.4-33.4)$ & .04 & $11.7(4.7-22.4)$ & $18.8(6.0-28.6)$ & .22 \\
\hline STS-PROM, \% median (IQR) & $4.9(2.5-12)$ & $8.0(2.5-14.3)$ & .44 & $4.1(1.7-6.7)$ & $5.2(2.6-10.8)$ & .49 \\
\hline eGFR $<60$ mL/kg/min, $\mathrm{n}(\%)$ & $72(64.3)$ & $25(58.1)$ & .48 & $27(57.5)$ & $15(71.4)$ & .27 \\
\hline $\mathrm{CAD}, \mathrm{n}(\%)$ & $82(73.2)$ & $28(65.1)$ & .32 & $15(31.9)$ & $7(33.3)$ & .91 \\
\hline COPD, n $(\%)$ & $24(21.4)$ & $13(30.2)$ & .25 & $10(21.3)$ & $7(33.3)$ & .29 \\
\hline CVD, n $(\%)$ & $9(8.0)$ & $6(13.9)$ & .27 & $2(4.3)$ & $4(19.0)$ & .05 \\
\hline Previous cardiac surgery, n (\%) & $31(27.7)$ & 17 (39.6) & .15 & $9(19.1)$ & $4(19.0)$ & .99 \\
\hline Atrial fibrillation, $\mathrm{n}(\%)$ & $32(28.6)$ & $18(41.9)$ & .11 & $19(40.4)$ & $11(52.4)$ & .36 \\
\hline CRT, n (\%) & $38(33.9)$ & $9(21.0)$ & .11 & $0(0)$ & $0(0)$ & - \\
\hline NYHA class III-IV, n (\%) & $89(79.5)$ & $33(76.8)$ & .71 & $29(61.7)$ & $13(61.9)$ & .99 \\
\hline Preoperative inotropes, n (\%) & $7(6.2)$ & $3(7.0)$ & .87 & $0(0)$ & $0(0)$ & - \\
\hline pro-BNP, pg/mL median (IQR) & $3163(1291-6036)$ & $3154(1419-9209)$ & .63 & $1023(455-1822)$ & $782(299-2774)$ & .47 \\
\hline \multicolumn{7}{|l|}{ Echocardiographic } \\
\hline $\mathrm{EDD}, \mathrm{mm}$ & $67.3 \pm 8.0$ & $70.2 \pm 7.8$ & .049 & $55.9 \pm 9.4$ & $59.0 \pm 7.0$ & .19 \\
\hline $\mathrm{ESD}, \mathrm{mm}$ & $53.7 \pm 8.8$ & $57.1 \pm 10.0$ & .057 & $36.8 \pm 9.3$ & $37.5 \pm 7.4$ & .77 \\
\hline $\mathrm{EF}, \%$ & $28.0 \pm 9.3$ & $28.8 \pm 9.9$ & .62 & $54.2 \pm 11.8$ & $63.3 \pm 9.5$ & $<.01$ \\
\hline EVEREST suitability & $47(41.9)$ & $16(37.2)$ & .59 & $24(51.0)$ & $15(71.4)$ & .12 \\
\hline $\mathrm{CD}, \mathrm{mm}$ & $12 \pm 3$ & $12 \pm 3$ & .96 & - & - & - \\
\hline $\mathrm{CL}, \mathrm{mm}$ & $3.6 \pm 1.5$ & $3.8 \pm 1.7$ & .62 & - & - & - \\
\hline $\mathrm{TA}, \mathrm{cm}^{2}$ & $2.6 \pm 0.9$ & $2.9 \pm 1.2$ & .21 & - & - & - \\
\hline IC jet extension, mm & $12 \pm 4$ & $13 \pm 4$ & .32 & $13.9 \pm 3.1$ & $11.7 \pm 2.9$ & .13 \\
\hline $\mathrm{FG}, \mathrm{mm}$ & - & - & - & $5.0 \pm 2.2$ & $4.6 \pm 1.5$ & .63 \\
\hline $\mathrm{FW}, \mathrm{mm}$ & - & - & - & $12.8 \pm 3.0$ & $12.8 \pm 1.5$ & .97 \\
\hline MVA, $\mathrm{cm}^{2}$ & $4.8 \pm 0.9$ & $5.2 \pm 1.5$ & .23 & $5.0 \pm 1.5$ & $6.5 \pm 2.1$ & .27 \\
\hline IC annular length, mm & $40 \pm 6$ & $40 \pm 5$ & .82 & $41.3 \pm 8.5$ & $43.8 \pm 8.8$ & .67 \\
\hline SL annular length, mm & $36 \pm 5$ & $39 \pm 5$ & .022 & $38.6 \pm 2.4$ & $38.8 \pm 5.7$ & .96 \\
\hline Annular calcifications & $2(1.8)$ & $0(0)$ & .38 & $5(10.6)$ & $1(4.8)$ & .43 \\
\hline Leaflet calcifications & $5(4.4)$ & $2(4.6)$ & .94 & $3(6.4)$ & $1(4.8)$ & .79 \\
\hline Leaflet cleft & $10(8.9)$ & $4(9.3)$ & .92 & $4(8.5)$ & $2(9.5)$ & .89 \\
\hline Multiple jets & $4(3.6)$ & $2(4.6)$ & .74 & $1(2.1)$ & $1(4.8)$ & .55 \\
\hline sPAP, mm Hg & $48 \pm 14$ & $56 \pm 17$ & .004 & $47 \pm 16$ & $47 \pm 16$ & .87 \\
\hline $\mathrm{TR} \geq 3+, \mathrm{n}(\%)$ & $26(23.2)$ & $19(44.2)$ & .01 & $12(25.5)$ & $4(19.0)$ & .56 \\
\hline $\mathrm{S}-\mathrm{TDI}, \mathrm{cm} / \mathrm{s}$ & $9.8 \pm 3.2$ & $9.9 \pm 3.3$ & .93 & $11.8 \pm 1.8$ & $11.8 \pm 1.6$ & .96 \\
\hline
\end{tabular}

FMR, Functional mitral regurgitation; DMR, degenerative mitral regurgitation; $M R$, mitral regurgitation; euroSCORE, European System for Cardiac Operative Risk Evaluation; $I Q R$, interquartile range; STS-PROM, Society of Thoracic Surgeons Predicted Mortality; $e G F R$, estimated glomerular filtrate rate; $C A D$, coronary artery disease; $C O P D$, chronic obstructive pulmonary disease; $C V D$, cerebrovascular disease; $C R T$, cardiac resynchronization therapy; $N Y H A$, New York Heart Association; $B N P$, brain natriuretic peptide; $E D D$, end-diastolic diameter; $E S D$, end-systolic diameter; $E F$, ejection fraction; $E V E R E S T$, Endovascular Valve Edge-to-Edge REpair Study; $C D$, coaptation depth; $C L$, coaptation length; $T A$, tented area; $I C$, intercommissural; $F G$, flail gap; $F W$, flail width; $M V A$, mitral valve area; $S L$, septum-lateral; $s P A P$, systolic pulmonary artery pressure; $T R$, tricuspid regurgitation; S-TDI, $\mathrm{S}$ wave at tissue Doppler imaging.

\section{Degenerative Mitral Regurgitation Group}

In DMR, 3 of 15 deaths $(20 \%)$ were cardiac related. MR $\geq 3+$ recurrence was observed in 12 of 68 patients $(17.6 \%)$. NYHA class III or greater was found in 7 of 68 patients $(10.3 \%)$.

CIF of MR $\geq 3+$ recurrence in patients with $\mathrm{MR} \leq 1+$ and MR $2+$ resulted in $6.9 \% \pm 4.0 \%$ and $43.4 \% \pm$ $10.1 \%$ at 24 months, respectively (Gray test $P=.003$ ). Regression modeling of the effect of covariates on CIF confirmed that residual MR $2+$ after the procedure was the only factor significantly related to the development of
MR $\geq 3+(\mathrm{HR}, 5.31 ; 95 \%$ CI, $1.63-17.2 ; P<.005)$. No further analysis is reported in the DMR group because of the small number of patients and events.

\section{DISCUSSION}

In this study, we found an unfavorable impact on followup outcomes of acute residual 2+ MR after MitraClip repair when compared with residual $\leq 1+$ MR. Toggweiler and colleagues $^{5}$ reported impaired short-term survival to be associated with an increasingly higher degree of residual MR after the MitraClip procedure. However, in this study, 


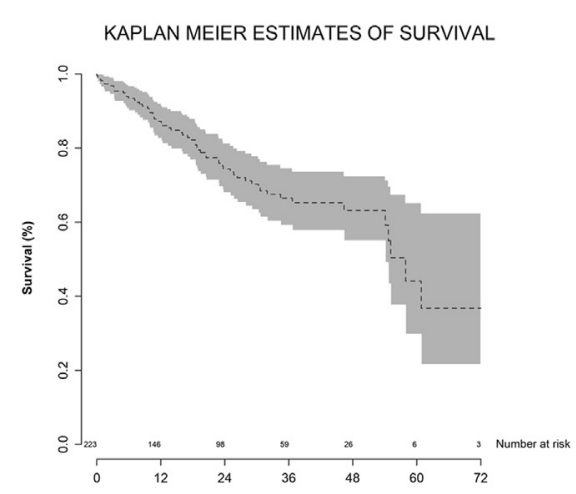

A

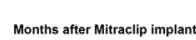

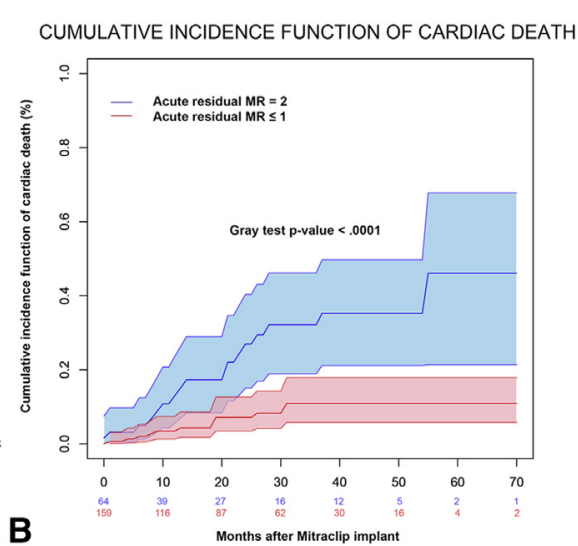

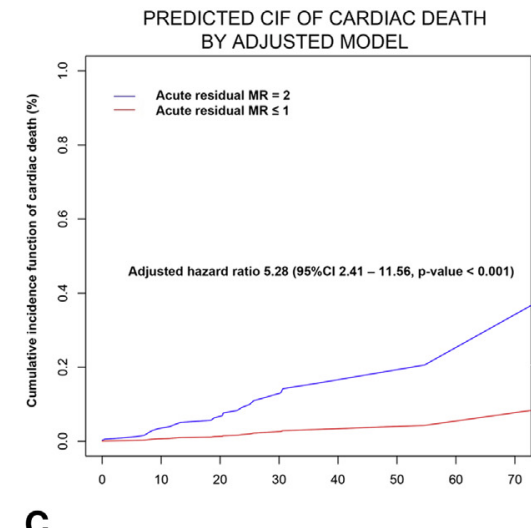

C

FIGURE 1. A, Unadjusted Kaplan-Meier estimates of long-term survival. B, Unadjusted CIF of cardiac death, with noncardiac death as competing risk. The CIF of patients with residual MR $2+$ was significantly higher compared with those with a residual MR $\leq 1+($ Gray test $P<.001$ ). C, Adjusted CIF of cardiac death, with noncardiac death as competing risk. $M R$, Mitral regurgitation; $C I F$, cumulative incidence function; $C I$, confidence interval.

there were only 31 patients with residual $2+$ MR (17 with FMR, 14 with DMR), and no specific outcome was reported for this group. Pooled data from the Endovascular Valve Edge-to-Edge REpair Study II High Risk study and the Real World Expanded Multi-center Study of the MitraClip System registry reported 1-year outcomes from a high-risk population with DMR, among whom 35 patients had residual $2+$ MR. In this experience, no difference was observed in survival or probability of NYHA I and II class between the $2+$ MR and the $\leq 1+$ MR group. Our study is the first to focus on the comparison of follow-up outcomes between patients with $2+$ and $\leq 1+$ MR, assessing both the FMR and DMR settings.

\section{Survival}

In patients with FMR, a significantly increased cardiac death rate was observed with residual $2+$ MR compared with $\leq 1+$ MR. This is in line with the well-known data from Grigioni and colleagues, ${ }^{19}$ who reported a graded relationship between MR severity and reduced survival for less than severe ischemic FMR.

Patients with $2+$ MR showed a baseline higher risk profile due to a slightly more advanced heart disease:

TABLE 3. Hazard ratios of the adjusted Fine and Gray model for cardiac death after MitraClip (Abbott Vascular Inc, Santa Clara, Calif) implant

\begin{tabular}{lccc}
\hline \multicolumn{1}{c}{ Variable } & HR & $\mathbf{9 5} \%$ CI & $\boldsymbol{P}$ value \\
\hline Residual MR 2+ & 5.28 & $2.41-11.56$ & $<.001$ \\
Preoperative end-diastolic diameter & 1.15 & $1.08-1.22$ & $<.001$ \\
Preoperative NYHA class IV & 2.29 & $1.01-5.22$ & .04 \\
eGFR $<60 \mathrm{~mL} / \mathrm{kg} / \mathrm{min}$ & 4.53 & $2.01-10.23$ & $<.001$ \\
Functional MR & 0.58 & $0.14-2.40$ & $.45^{*}$ \\
\hline
\end{tabular}

$H R$, Hazard ratio; $C I$, confidence interval; $M R$, mitral regurgitation; $N Y H A$, New York Heart Association; $e G F R$, estimated glomerular filtration rate. $* P>.05$. compared with the $\leq 1+$ MR group, they had larger ventricles with larger mitral valves and higher pulmonary pressure. Of note, these features may have impaired the surgeon's ability to achieve acute optimal MR reduction during the MitraClip procedure. Advanced left ventricle remodeling was a strong independent predictor of increased cardiac death. This association between the health of the left ventricle and impaired survival is easily understandable and consistent with data from the previous surgical experience. ${ }^{20}$

On the other hand, nothing can be said about the predictors of death due to cardiac reasons in patients affected by DMR because cardiac death occurred in only 3 of these patients in our series. Patients with DMR are usually elderly, frail, and affected by several comorbidities. These features, rather than their heart disease (which usually does not include advanced left ventricle dilatation or dysfunction), explain their high surgical risk and are likely to significantly impair their overall survival. Of note, Lim and colleagues ${ }^{8}$ did not find a significant difference between 1-year survival of patients with acute residual $2+$ MR compared with patients with $\leq 1+$ MR $(80.0 \%$ vs $83.3 \%$, respectively). These initial findings suggest that $2+$ residual MR may not be a big issue in regard to survival in such an old and complex patient population, in whom expected survival is limited and linked to several other factors.

Procedural failure (residual significant MR) is emerging as the most consistent predictor of poor outcomes after transcatheter mitral repair. When more data from patients without residual significant MR will be collected, a scoring system combining all other unfavorable parameters to predict long-term benefit or procedural futility could be evaluated. Unfortunately, reliable preoperative prediction of procedural success/failure remains an even more urgent issue to be solved. 

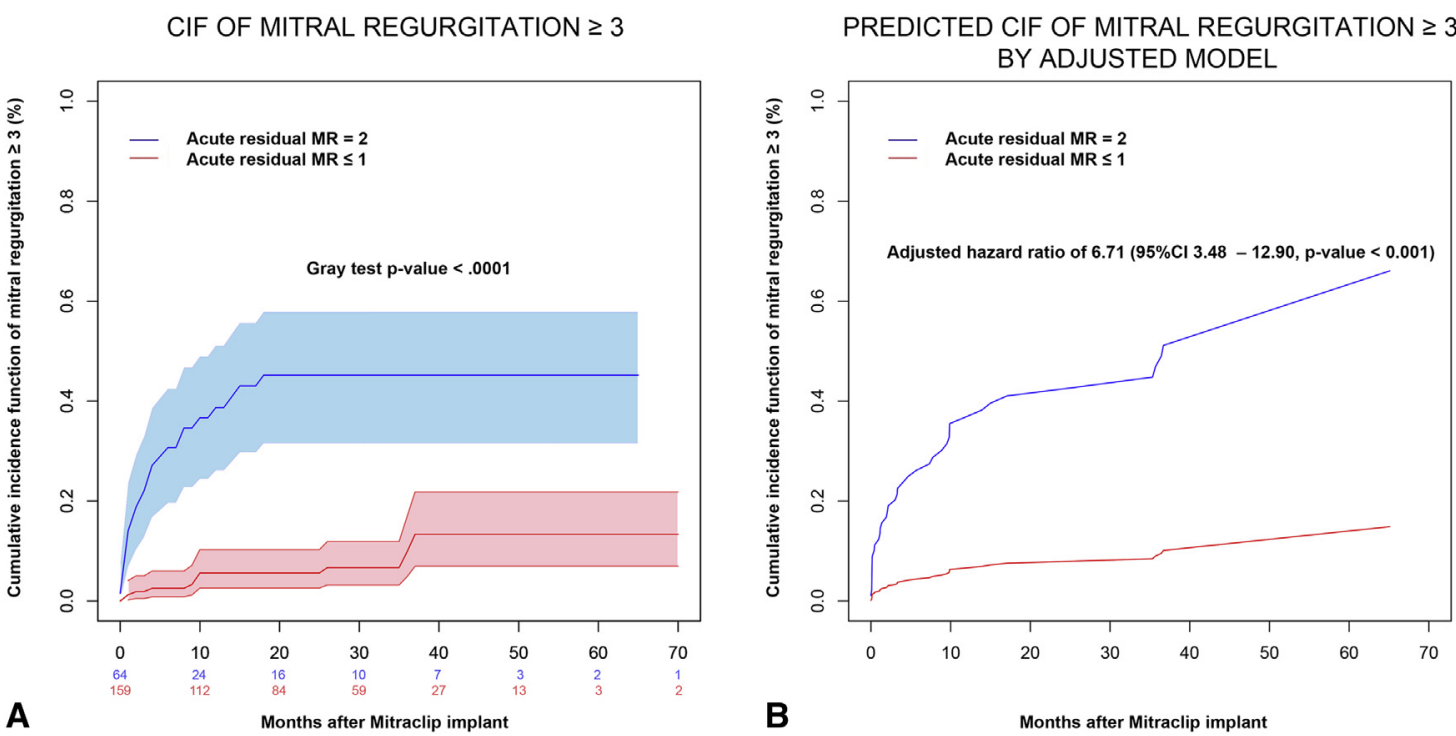

FIGURE 2. A, Unadjusted CIF of recurrent $M R \geq 3+$, with noncardiac death as competing risk. The CIF of patients with residual MR $2+$ was significantly higher compared with those with a residual $\mathrm{MR} \leq 1+($ Gray test $P<.001)$. B, Adjusted CIF of recurrent $\mathrm{MR} \geq 3+$, with noncardiac death as competing risk. $C I F$, Cumulative incidence function; $M R$, mitral regurgitation; $C I$, confidence interval.

\section{Mitral Regurgitation Recurrence}

MR recurrence was remarkably higher in the $2+$ MR group compared with the $\leq 1+$ MR group, in both the FMR and DMR groups. Of note, it was also quick to develop, with $21.4 \%$ of cases of progression occurring during the first month after the procedure in FMR. This poor efficacy in a population of patients who were supposed to have had a "procedural success" is striking.

It must be remembered however that in the setting of FMR durable results are also challenging to achieve with surgery, with early repair failure observed after ring annuloplasty in up to $25 \%$ of patients after 6 months. ${ }^{21}$ We know this is because FMR requires a perfect anatomic selection, and, most important, it is actually not just a mitral disease but a ventricular disease. ${ }^{22}$ For these reasons, a late mitral repair, when left ventricle remodeling is already too advanced, is most frequently going to fail. ${ }^{20,23}$ FMR is per se a dynamic and variable entity that strongly depends on left ventricle geometry and function but also on pressure and volume loads. In this regard, we also want to remember that general anesthesia and inotropic support can substantially reduce MR. ${ }^{24-26}$ Because it is well

TABLE 4. Generalized mixed effect model predicting postoperative New York Heart Association class (treated as ordinal data)

\begin{tabular}{lccc}
\hline \multicolumn{1}{c}{ Variable } & OR & $\mathbf{9 5} \%$ CI & $\boldsymbol{P}$ value \\
\hline Residual MR 2+ & 1.25 & $0.98-1.59$ & .07 \\
Preoperative NYHA & 1.30 & $1.08-1.56$ & .006 \\
eGFR $<60 \mathrm{~mL} / \mathrm{kg} / \mathrm{min}$ & 1.35 & $1.07-1.70$ & .01 \\
\hline
\end{tabular}

Random effect of intercept: $0.16 \pm 0.40 . O R$, Odds ratio; $C I$, confidence interval; $M R$, mitral regurgitation; NYHA, New York Heart Association; eGFR, estimated glomerular filtration rate. recognized that intraoperative MR underestimation can play a role in high FMR recurrence, this should be taken into account in the operating room at the end of the procedure when assessing the final MR degree. Maneuvers such as the Trendelenburg volume stress test may be useful to exacerbate a possible latent significant MR.

In DMR, the high rate of MR recurrence in the patients with $2+$ MR has long been anticipated, because it was repeatedly reported in long-term studies on the surgical edge-to-edge technique. ${ }^{10,27}$ Even more important, longterm surgical results of the edge-to-edge repair without concomitant annuloplasty (as with the MitraClip) have shown poor follow-up freedom from MR recurrence in DMR ( $43 \%$ at 12 years), $2+$ MR being once again a significant predictor of MR recurrence. ${ }^{28}$ Of note, in that series, freedom from $M R \geq 3+$ recurrence in patients with residual $\leq 1+$ MR was $80 \%$ at 5 years. By comparison, freedom from MR $\geq 3+$ in our study in this group of patients was $84 \%$ after 4 years and $84 \%$ after 3 years in FMR and DMR, respectively.

These data of patients with "real" procedural success can be judged as promising given their high-risk profile and should inspire us to seek a better preoperative understanding of patient selection and pursue the best possible result in the operating room. On the other hand, no matter how much we try, transcatheter mitral repair technologies must reach a better efficacy profile, especially before expanding indications of these procedures to mid- or even low-risk patients. The current intrinsic design of transcatheter mitral edge-to-edge device, used alone, will hardly be able to achieve optimal MR reduction in all patients, especially 
CIF OF CARDIAC DEATH IN FUNCTIONAL MITRAL REGURGITATIATION

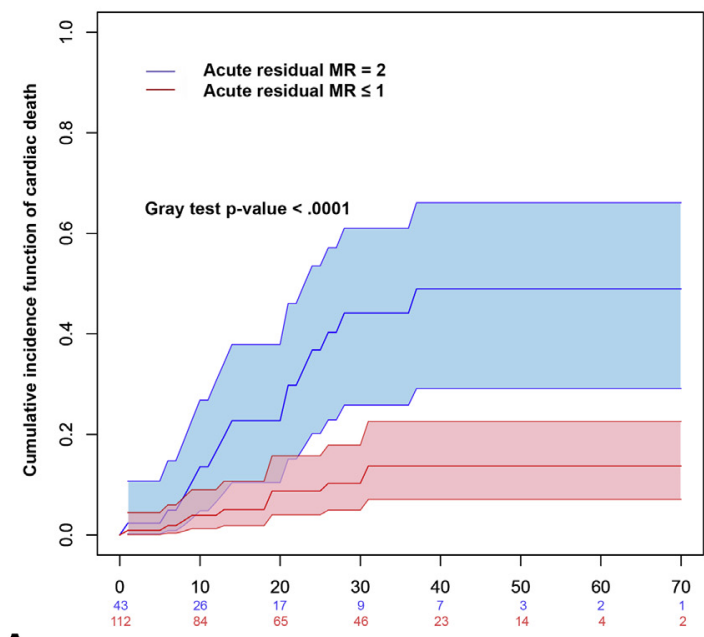

A

FIGURE 3. A, Unadjusted CIF of cardiac death in functional MR cause, with noncardiac death as competing risk. The CIF of patients with residual MR $2+$ was significantly higher compared with those with a residual MR $\leq 1+$ (Gray test $P<.001$ ). B, Unadjusted CIF of recurrent MR $\geq 3+$ in functional MR cause, with noncardiac death as competing risk. The CIF of patients with residual MR $2+$ was significantly higher compared with those with a residual MR $\leq 1+$ (Gray test $P<.001)$. CIF, Cumulative incidence function; $M R$, mitral regurgitation.

in case of complex leaflet pathology and concurrent annular dilation. Because annuloplasty has already proved to be fundamental to obtain durable repair results in surgery in both FMR $^{29-31}$ and DMR, ${ }^{32,33}$ the concomitant use of transcatheter mitral annuloplasty devices, which are now arriving on the scene, ${ }^{34}$ may enable a big improvement in the overall efficacy of percutaneous mitral repair in the next years.

We acknowledge using more clips per patient (1.75 clip per patient) in our center than most other centers do. More clips were actually used in patients who ended up with residual $2+$ MR, likely because of their more difficult anatomy or suboptimal clip implantation with leaflet distortion and residual MR. On the other hand, although we used more than 1 clip in more than $60 \%$ of patients with $\leq 1+\mathrm{MR}$, more than 2 clips were rarely necessary to achieve an optimal result.

In our series, $5.8 \%$ of patients required some kind of mitral reintervention (10 surgeries, 3 MitraClip reimplantations). Close follow-up is mandatory. MR severity, symptoms, and risk of surgery must be balanced to find the optimal timing for reoperation. Relatively "lower" risk would lean toward prompt intervention to avoid further worsening of heart and other organ function and to avoid repeated heart decompensations, each of which can be deadly in these patients. Of note, although we do not have a large experience, the MitraClip can sometimes help bridge a decompensated patient to LVAD/transplantation. We believe that the indications to such therapies remain the same as in patients without the MitraClip, mainly involving symptoms, right ventricle function, and renal function.
CIF OF MR $\geq 3$ IN FUNCTIONAL MITRAL REGURGITATIATION

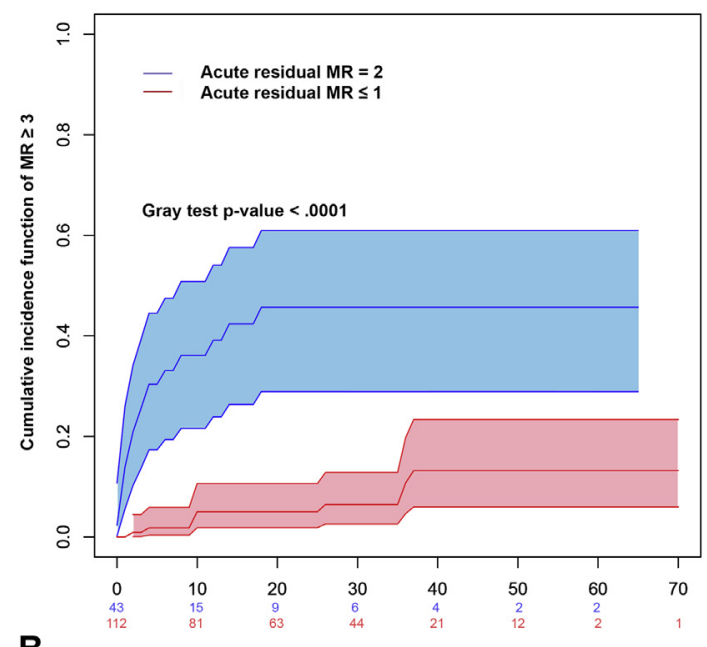

B

\section{Study Limitations}

The major limitation of this study is the small number of patients, which did not allow some subgroup analyses, particularly the clinical outcomes prediction in the DMR group and the prediction of MR worsening in the residual $2+$ MR group. Moreover, the follow-up time was still limited. Furthermore, because even the first patients in our experience were included in the study, a learning curve may have played a role (eg, some early partial clip detachment). Also, it must be remembered that the assessment of residual MR in the setting of a double-orifice valve remains a challenging task in terms of both raw regurgitation quantification and physiopathologic significance. Indeed, most quantitative and qualitative echocardiographic methods have been designed to assess the degree of regurgitation in a single regurgitant orifice, and unreliable estimation of MR has been described in the double-orifice setting. ${ }^{35}$

\section{CONCLUSIONS}

Residual 2+ MR after MitraClip repair was associated with worse follow-up outcomes compared with $\leq 1+$ MR, including survival, symptom relief, and MR recurrence. Better efficacy should be pursued by transcatheter mitral repair technologies before they can be expanded to intermediate- or low-risk patients.

\section{Conflict of Interest Statement}

N.B. is a consultant for Abbott Vascular. P.D. is a consultant for Abbott Vascular. F.B. reports receiving consulting fees 
from St Jude Medical. All other authors have nothing to disclose with regard to commercial support.

\section{References}

1. Vahanian A, Alfieri O, Andreotti F, Antunes MJ, Baron-Esquivias G, Baumgartner $\mathrm{H}$, et al. Guidelines on the management of valvular heart disease (version 2012): The Joint Task Force on the Management of Valvular Heart Disease of the European Society of Cardiology (ESC) and the European Association for Cardio-Thoracic Surgery (EACTS). Eur J Cardiothorac Surg. 2012;42:S1-44.

2. Mirabel M, Iung B, Baron G, Messika-Zeitoun D, Detaint D, Vanoverschelde JL, et al. What are the characteristics of patients with severe, symptomatic, mitral regurgitation who are denied surgery? Eur Heart J. 2007;28:1358-65.

3. Maisano F, Franzen O, Baldus S, Schafer U, Hausleiter J, Butter C, et al. Percutaneous mitral valve interventions in the real world: early and 1 -year results from the ACCESS-EU, a prospective, multicenter, nonrandomized post-approval study of the MitraClip therapy in Europe. J Am Coll Cardiol. 2013;62:1052-61.

4. Baldus S, Schillinger W, Franzen O, Bekeredjian R, Sievert H, Schofer J, et al. MitraClip therapy in daily clinical practice: initial results from the German transcatheter mitral valve interventions (TRAMI) registry. Eur J Heart Fail. 2012; $14: 1050-5$.

5. Toggweiler S, Zuber M, Surder D, Biaggi P, Gstrein C, Moccetti T, et al. Two-year outcomes after percutaneous mitral valve repair with the MitraClip system: durability of the procedure and predictors of outcome. Open Heart. 2014; 1:e000056.

6. Puls M, Tichelbacker T, Bleckmann A, Hunlich M, von der Ehe K, Beuthner BE, et al. Failure of acute procedural success predicts adverse outcome after percutaneous edge-to-edge mitral valve repair with MitraClip. Eurointervention. 2014;9:1407-17.

7. Paranskaya L, D'Ancona G, Bozdag-Turan I, Akin I, Kische S, Turan GR, et al. Residual mitral valve regurgitation after percutaneous mitral valve repair with the MitraClip(R) system is a risk factor for adverse one-year outcome. Catheter Cardiovasc Interv. 2013;81:609-17.

8. Lim DS, Reynolds MR, Feldman T, Kar S, Herrmann HC, Wang A, et al, Improved functional status and quality of life in prohibitive surgical risk patients with degenerative mitral regurgitation after transcatheter mitral valve repair. $J$ Am Coll Cardiol. 2014;64:182-92.

9. Glower DD, Kar S, Trento A, Lim DS, Bajwa T, Quesada R, et al. Percutaneous mitral valve repair for mitral regurgitation in high-risk patients: results of the EVEREST II study. J Am Coll Cardiol. 2014;64:172-81.

10. De Bonis M, Lapenna E, Lorusso R, Buzzatti N, Gelsomino S, Taramasso M, et al. Very long-term results (up to 17 years) with the double-orifice mitral valve repair combined with ring annuloplasty for degenerative mitral regurgitation. $J$ Thorac Cardiovasc Surg. 2012;144:1019-24.

11. Mauri L, Garg P, Massaro JM, Foster E, Glower D, Mehoudar P, et al. The EVEREST II Trial: Design and rationale for a randomized study of the evalve mitraclip system compared with mitral valve surgery for mitral regurgitation. Am Heart J. 2010;160:23-9.

12. Zoghbi WA, Enriquez-Sarano M, Foster E, Grayburn PA, Kraft CD, Levine RA, et al. Recommendations for evaluation of the severity of native valvular regurgitation with two-dimensional and Doppler echocardiography. J Am Soc Echocardiogr. 2003;16:777-802.

13. Putter H, Fiocco M, Geskus RB. Tutorial in biostatistics: competing risks and multi-state models. Stat Med. 2007;26:2389-430.

14. Barili F, Barzaghi N, Cheema FH, Capo A, Jiang J, Ardemagni E, et al. An original model to predict intensive care unit length-of stay after cardiac surgery in a competing risk framework. Int J Cardiol. 2013;168:219-25.

15. Pintilie M. Competing Risks: A Practical Perspective; 2006. John Wiley \& Sons, Ltd: Chichester, UK.

16. Berridge DM. Multivariate Generalized Linear Mixed Models Using R; 2011. CRC Press, Taylor \& Francis Group, LLC: Boca Raton, FL.

17. Janssen KJ, Donders AR, Harrell FE Jr, Vergouwe Y, Chen Q, Grobbee DE, et al. Missing covariate data in medical research: to impute is better than to ignore. $J$ Clin Epidemiol. 2010;63:721-7.

18. Harrell FEJ. Regression Modeling Strategies; 2001. Springer New York: New York, NY.

19. Grigioni F, Enriquez-Sarano M, Zehr KJ, Bailey KR, Tajik AJ. Ischemic mitral regurgitation: long-term outcome and prognostic implications with quantitative Doppler assessment. Circulation. 2001;103:1759-64.
20. Braun J, de Veire NR, Klautz RJM, Versteegh MIM, Holman ER, Westenberg JJM, et al. Restrictive mitral annuloplasty cures ischemic mitral regurgitation and heart failure. Ann Thorac Surg. 2008;85:430-7.

21. McGee EC, Gillinov AM, Blackstone EH, Rajeswaran J, Cohen G, Najam G et al. Recurrent mitral regurgitation after annuloplasty for functional ischemic mitral regurgitation. J Thorac Cardiovasc Surg. 2004;128:916-24.

22. Levine RA, Schwammenthal E. Ischemic mitral regurgitation on the threshold of a solution: from paradoxes to unifying concepts. Circulation. 2005;112: 745-58.

23. De Bonis M, Lapenna E, Verzini A, La Canna G, Grimaldi A, Torracca L, et al Recurrence of mitral regurgitation parallels the absence of left ventricular reverse remodeling after mitral repair in advanced dilated cardiomyopathy. Ann Thorac Surg. 2008;85:932-9.

24. Sheikh KH, Bengtson JR, Rankin JS, Debruijn NP, Kisslo J. Intraoperative transesophageal Doppler color flow imaging used to guide patient selection and operative treatment of ischemic mitral regurgitation. Circulation. 1991;84: 594-604.

25. Bach DS, Deeb GM, Bolling SF. Accuracy of intraoperative transesophageal echocardiography for estimating the severity of functional mitral regurgitation. Am J Cardiol. 1995;76:508-12.

26. Aklog L, Filsoufi F, Flores KQ, Chen RH, Cohn LH, Nathan NS, et al. Does coronary artery bypass grafting alone correct moderate ischemic mitral regurgitation? Circulation. 2001;104:168-75.

27. De Bonis M, Lapenna E, Taramasso M, La Canna G, Buzzatti N, Pappalardo F, et al. Very long-term durability of the edge-to-edge repair for isolated anterior mitral leaflet prolapse: up to 21 years of clinical and echocardiographic results. J Thorac Cardiovasc Surg. 2014;148:2027-32.

28. De Bonis M, Lapenna E, Maisano F, Barili F, La Canna G, Buzzatti N, et al Long-term results ( $</=18$ years) of the edge-to-edge mitral valve repair without annuloplasty in degenerative mitral regurgitation: implications for the percutaneous approach. Circulation. 2014;130:S19-24.

29. Bolling SF, Pagani FD, Deeb GM, Bach DS. Intermediate-term outcome of mitral reconstruction in cardiomyopathy. J Thorac Cardiovasc Surg. 1998;115:381-6.

30. Tibayan FA, Rodriguez F, Langer F, Zasio MK, Bailey L, Liang D, et al. Annular remodeling in chronic ischemic mitral regurgitation: ring selection implications. Ann Thorac Surg. 2003;76:1549-54.

31. Kwon MH, Lee LS, Cevasco M, Couper GS, Shekar PS, Cohn LH, et al Recurrence of mitral regurgitation after partial versus complete mitral valve ring annuloplasty for functional mitral regurgitation. J Thorac Cardiovasc Surg. 2013;146:616-22.

32. Flameng W, Herijgers P, Bogaerts K. Recurrence of mitral valve regurgitation after mitral valve repair in degenerative valve disease. Circulation. 2003;107: $1609-13$.

33. Gillinov AM, Cosgrove DM, Blackstone EH, Diaz R, Arnold JH, Lytle BW, et al Durability of mitral valve repair for degenerative disease. J Thorac Cardiovasc Surg. 1998;116:734-43.

34. Maisano F, La Canna G, Latib A, Denti P, Taramasso M, Kuck KH, et al First-in-man trans-septal implantation of a "surgical-like" mitral valve annuloplasty device for functional mitral regurgitation. JACC Cardiovasc Interv. 2014;7:1326-8.

35. Lin BA, Forouhar AS, Pahlevan NM, Anastassiou CA, Grayburn PA, Thomas JD et al. Color Doppler jet area overestimates regurgitant volume when multiple jets are present. J Am Soc Echocardiogr. 2010;23:993-1000.

Key Words: MitraClip, mitral regurgitation, transcatheter, repair

\section{Discussion}

Dr M. Ruel (Ottawa, ON, Canada). Over the years, we have been impressed by the dedication to the scientific understanding of their edge-to-edge mitral repair operation that Buzzatti and colleagues have displayed. Today, they are again to be commended for investigating when and why the concept that they have invented does not always work, whether in the surgical or the transcatheter fashion, which 
in my opinion requires scientific humility and is an example for all of us to follow.

As we have just heard, patients who underwent a MitraClip procedure with a "procedural success" of $2+$ residual MR do not do well from a survival, functional, and echocardiographic point of view, particularly if their MR was of a functional nature. This is a new and important finding, because as was mentioned earlier, procedural success with the MitraClip includes patients who have residual 2+ MR. This helps us understand the prognosis and may guide therapy in those patients. Of note is that the same poor prognostic implications were not seen if the same 2+ residual MR was observed in patients whose MR was due to degenerative disease and not functional disease.

Dr Buzzatti, how many clips were used, and for a given degree of ventricular dilatation and posterior leaflet angle on the mitral valve, did the number of clips correlate with freedom from adverse events? In other words, do you believe that patients with $2+$ residual MR were treated as optimally as they could have been? It is approximately 10 years now since the MitraClip has taken off, so I think considering the current experience and expertise that we have with this procedure, do you think all patients were treated optimally?

Dr Buzzatti. We now tend to use more clips than most centers do, because we want to optimize our final result. In FMR, we observed an association between the larger ventricles and the use of a larger number of clips, although this was not associated with survival or other outcomes. Unfortunately, if you have a big ventricle, just putting more clips will not be enough to make your patient fine.

Over time, we changed our way to do this procedure, implanting more clips to treat not just the jet, not just the MR, but also the lesion. This is particularly important in the DMR subgroup. We had patients in whom we put 1 clip in the early years, and the jet basically went away with just trace residual MR, but we still had residual prolapse.

I do not have strong data to support this, but in our experience when you still see prolapse, you should go for another clip, even if you don't have significant residual MR, to correct the mitral lesion to avoid partial detachment, which can happen very early after the procedure in such cases.

Dr Ruel. Assuming that most patients were treated with the best of currently available technology and techniques, can you suggest a scoring system or perhaps an unfavorable combination or constellation of patient factors such as tricuspid regurgitation? We must remember in your series that at least 3+ TR was found in approximately half of the patients with FMR who had 2+ residual MR. Other undesirable factors may be ventricular size and pulmonary artery pressures, and certainly these have been shown in other articles to be unfavorable factors with both surgical and transcatheter mitral valve repair for FMR. Do you think there is a constellation of such undesirable factors that would translate into an attempt at the MitraClip being futile or, at the very least, not cost-effective?

Dr Buzzatti. Probably, but I don't know which it is. Yes, as you said, all these factors, tricuspid regurgitation, a large annulus diameter, and poor ejection fraction, indicate a more advanced stage of the disease, and if the disease is too advanced, our MitraClip is going to be a failure sooner or later.

In our experience, what we look for as the most important parameter is the left ventricular end-diastolic diameter. Systolic pulmonary artery pressure and tricuspid regurgitation sometimes get better after the MitraClip, and we do not know yet which patients will improve and which will not. So the left ventricle is the one to look for, at the least, now.

Dr Ruel. You showed us that $2+$ residual MR with DMR is probably okay or at least not associated with adverse clinical events in the present small subseries that you showed us. However, with functional MR, we have alternatives, such as mitral valve replacement and destination LVAD. These are always a consideration.

How long do you think one should wait before proposing more invasive advanced strategies in patients with FMR who are left with residual 2+ MR before they are too far gone or cardiac death occurs? Do you have any follow-up rescue therapy type of experience to report within the present series that you showed us today?

Dr Buzzatti. Yes, we do have some patients in whom we implanted an LVAD as destination therapy after the MitraClip, 3 patients, actually, and another 2 patients whom we treated with the MitraClip ended up with an LVAD in another center. I think the indications are the same as for other patients who were not treated with a MitraClip. You see if the patient is symptomatic, if the right ventricle is okay, and the creatinine is fine and so on. The MitraClip can help you to put a patch in the patient who is decompensated in the intensive care unit and you cannot get him out. So you may put a clip, reduce MR, and let him improve just a little bit, take time and reevaluate him, to see if the right ventricle recovers and so on.

Dr Ruel. Congratulations again on an excellent presentation, and I thank the Association for the privilege of discussing this important article. 